\title{
Capacitação dos profissionais de enfermagem frente às situações de urgência e emergência na atenção primária a saúde: revisão integrativa
}

\author{
Training of nursing professionals facing urgency and emergency situations in primary health care: \\ integrative review \\ La formación de los profesionales de enfermería ante las situaciones de urgencia y emergencia en \\ atención primaria: revisión integradora
}

Recebido: 12/09/2021 | Revisado: 19/09/2021 | Aceito: 24/09/2021 | Publicado: 26/09/2021

Lorena Esmeralda Nascimento Celeste

ORCID: https://orcid.org/0000-0001-8217-620X Universidade Salvador, Brasil

E-mail: lory.celeste@yahoo.com.br

Maiara Rodrigues Maia

ORCID: https://orcid.org/0000-0002-6719-2911 Hospital Sírio Libanês, Brasil

E-mail: maiara.enfermagem@ @otmail.com

Viviane Almeida Andrade

ORCID: https://orcid.org/0000-0003-3884-3719 Aquarela Clínica de Desenvolvimento Infantil e Terapia ABA, Brasil

E-mail: professora.viviane@yahoo.com.br

\begin{abstract}
Resumo
Objetivo: Identificar os requisitos para capacitação da equipe de enfermagem para atuar nas situações de urgência e emergência na Atenção Primaria à Saúde. Metodologia: Trata-se de uma revisão integrativa, estruturada conforme a metodologia PRISMA. A pesquisa teve como cenário uma Unidade de Saúde da Família, localizada em um bairro do Subúrbio Ferroviário de Salvador/Ba, participaram da pesquisa três Enfermeiros e cinco Técnicos de Enfermagem, os dados foram obtidos através de um questionário, por meio de um aplicativo de mensagens instantâneas para smartphone. Resultados: A amostra foi composta por profissionais de enfermagem, todos os participantes declararam que já trabalharam em emergência, destes apenas 75\% foram capacitados e somente 37,5\% qualificados pela Secretaria Municipal de Saúde e 50\% se sentem aptos para atuar nos atendimentos de emergência, nenhum participante utiliza a Escala de Manchester durante os atendimentos de urgência e emergência. Com essa análise percebe-se a falta de segurança dos profissionais e a carência da prática e experiência envolvendo as situações de emergência. Considerações finais: É imprescindível a realização de cursos de capacitação para todos os profissionais de enfermagem da APS, visto que a falta de informação sobre a temática pode afetar a assistência recomendada pela atenção primária.
\end{abstract}

Palavras-chave: Emergência; Atenção primária à saúde; Capacitação; Profissionais de enfermagem.

\begin{abstract}
Objective: To identify the requirements for training the nursing team to act in urgent and emergency situations in Primary Health Care. Methodology: This is an integrative review, structured according to the PRISMA methodology. The research had as scenario a Family Health Unit, located in a neighborhood of the Railway Suburb of Salvador/Ba, participated in the research three Nurses and five Nursing Technicians, data were obtained through a questionnaire, through an instant messaging application for smartphone. Results: The sample was composed of nursing professionals, all participants stated that they have worked in emergency, of these only $75 \%$ were trained and only $37.5 \%$ qualified by the Municipal Health Secretariat and 50\% feel able to act in emergency care, no participant uses the Manchester Scale during urgent and emergency care. With this analysis we can see the lack of security of professionals and the lack of practice and experience involving emergency situations. Final considerations: Training courses for all PHC nursing professionals are essential, since the lack of information on the subject can affect the assistance recommended by primary care.
\end{abstract}

Keywords: Emergency; Primary health care; Training; Nursing professionals.

\section{Resumen}

Objetivo: Identificar las necesidades de formación del equipo de enfermería para actuar en situaciones de urgencia y emergencia en Atención Primaria. Metodología: Se trata de una revisión integradora, estructurada según la metodología PRISMA. La investigación tuvo como escenario una Unidad de Salud Familiar, ubicada en un barrio del 
Suburbio Ferroviario de Salvador/Ba, participaron en la investigación tres Enfermeras y cinco Técnicos de Enfermería, los datos fueron obtenidos a través de un cuestionario, mediante una aplicación de mensajería instantánea para smartphone. Resultados: La muestra estuvo compuesta por profesionales de enfermería, todos los participantes manifestaron haber trabajado en emergencia, de estos solo el 75\% fueron capacitados y solo el 37,5\% calificados por la Secretaría Municipal de Salud y el 50\% se sienten capacitados para actuar en la atención de emergencia, ningún participante utiliza la Escala de Manchester durante la atención de urgencia y emergencia. Con este análisis podemos ver la falta de seguridad de los profesionales y la falta de práctica y experiencia en situaciones de emergencia. Consideraciones finales: Es imprescindible realizar cursos de formación para todos los profesionales de enfermería de la APS, ya que la falta de información sobre el tema puede afectar a la asistencia recomendada por la atención primaria.

Palabras clave: Emergencia; Atención primaria a la salud; Capacitación; Profesionales de la enfermería.

\section{Introdução}

A Atenção Primária a Saúde (APS) é a porta de entrada prioritária do SUS, pois evidencia a resolutividade dos cuidados primários diante dos problemas mais comuns de saúde da população. Caracteriza-se como uma atenção ambulatorial não especializada, tendo seus serviços ofertados pelas unidades de saúde habilitadas para apresentar condutas resolutivas (Silveira, 2020).

A Rede de Atenção a Saúde (RAS) é um conjunto dos serviços de saúde, organizado de forma hierárquica, articulado de maneira complementar e não competitiva, na perspectiva de redes de atenção, que sejam capazes de responder às necessidades de todos e de cada um, de maneira singular, integral, equânime e compartilhada. Sendo assim a APS responsável pelo cuidado e pelos atendimentos de demanda espontânea com base no protocolo de Acolhimento com Classificação de Risco (ACCR) (Damaceno, Lima, Pucci, \& Weiller, 2020).

Reformulada em 2017, a Política Nacional da Atenção Básica (PNAB) enfatiza que a APS é a principal porta de entrada da RAS, sendo responsável pelo primeiro atendimento nas situações de urgência e emergência mediante o ACCR, com a equipe qualificada para reconhecer tais situações, fornecendo suporte adequado até que sejam referenciadas para outros pontos de atenção da RAS quando necessário (Coloni, 2019).

O Sistema de Triagem de Manchester (STM) é a escala mais usada no ACCR, pois estabelece padrões de atendimento para as situações de urgência e emergência, definindo a ordem de atendimento de acordo com a gravidade. Além de ordenar o fluxo de atendimento, facilita nos critérios de priorização e implementa o princípio da Equidade no SUS (Moreira, Tibães, Batista, Cardoso, \& Brito, 2017).

A equipe de enfermagem da APS deve estar preparada para prestar cuidados necessários à população, entre suas atribuições aponta-se a necessidade de estar atento para complicações clínicas, que demandam ações de caráter emergencial ou de urgência, afinal de contas, a APS deve ser a primeira escolha para resolução de instabilidades clínicas e a equipe de saúde deve estar preparada para reconhecê-las, estabilizá-las e/ou referenciá-las, quando necessário (Nobrega, Bezerra, \& Sousa, 2015).

Produzido pelo Ministério da Saúde (2004), o documento Humaniza SUS, reconhece que os profissionais da atenção básica têm dificuldades de realizar atendimento ao paciente que chega à unidade com uma urgência de maior complexidade (Brasil, 2004).

Percebe-se, portanto a importância da capacitação contínua da equipe de enfermagem, aprimorando as ações voltadas para as situações de urgência e emergência. Considerando as informações apresentadas, as dificuldades dos profissionais de enfermagem nos atendimentos de urgência e emergência na Atenção Primária à Saúde, com ênfase na falta de capacitação dos profissionais de enfermagem, dificultando a identificação e resolubilidade dos atendimentos, justifica-se diante disso, o questionamento: quais os requisitos para capacitação da equipe de enfermagem para atuar nas situações de urgência e emergência na Atenção Primaria à Saúde? 
Para responder ao questionamento, o artigo tem como objetivo identificar os requisitos para capacitação da equipe de enfermagem para atuar nas situações de urgência e emergência na Atenção Primaria à Saúde.

\section{Metodologia}

Trata-se de uma pesquisa descritiva de abordagem quantitativa e qualitativa. O estudo tem como base uma revisão integrativa da literatura, foi realizado uma análise de dados, mediante a busca de artigos publicados no período de 2015 a 2020 , em periódicos científicos nacionais e internacionais com artigos relevantes a temática, disponíveis na Biblioteca Virtual em Saúde (BVS), Literatura Latino Americana e Ciências do Caribe (LILACS), Base de Dados em Enfermagem (BDENF) e Scientific Electronic Library Online (SCIELO). Como palavras chaves buscou-se estabelecer com base na plataforma de Descritores em Ciências da Saúde (DECS): Emergência, Enfermagem, Atenção Primária à Saúde, para complementar a base de estudos.

Para a revisão, foram estabelecidas as palavras chaves com base na plataforma de Descritores em Ciências da Saúde (DECS): Emergência, Enfermagem, Atenção Primária à Saúde. De acordo com os critérios de inclusão, os artigos deveriam estar disponíveis para leitura na íntegra, norteados ao objetivo de estudo, com resumos disponíveis nas bases de dados publicados entre 2015 a 2020. Quanto aos critérios de exclusão: artigos publicados em período que precede o ano de 2015 e os que não abordaram diretamente $\mathrm{o}$ tema proposto da pesquisa.

O estudo teve como cenário uma Unidade de Saúde da Família, situada no Subúrbio Ferroviário de Salvador, Bahia. A escolha do cenário se deu pela atuação da unidade de saúde no atendimento de acolhimento de demanda espontânea. Participaram do estudo 03 Enfermeiros e 05 Técnicos de Enfermagem. Os critérios de inclusão foram: ser profissional de enfermagem, estar trabalhando no momento da entrevista e aceitar participar do estudo voluntariamente.

Para a coleta de dados foi aplicado um questionário através do Google Forms, com questões fechadas, e o convite foi feito através de um aplicativo de mensagens instantâneas para smartphone e foi realizada nos meses de janeiro e fevereiro de 2021. Oroteiro teve as seguintes questões norteadoras: gênero, idade, escolaridade, tempo de atuação na APS, se já trabalhou em emergência, cursos de capacitação, Escala de Manchester e Acolhimento de demanda espontânea.

O processo de identificação, triagem, seleção e inclusão estão expostos no fluxograma PRISMA (Figura 1) que corrobora a totalidade das pesquisas bibliográficas e a técnica de seleção dos estudos. 
Figura 1 - Fluxograma da técnica de busca e seleção, Salvador 2021.

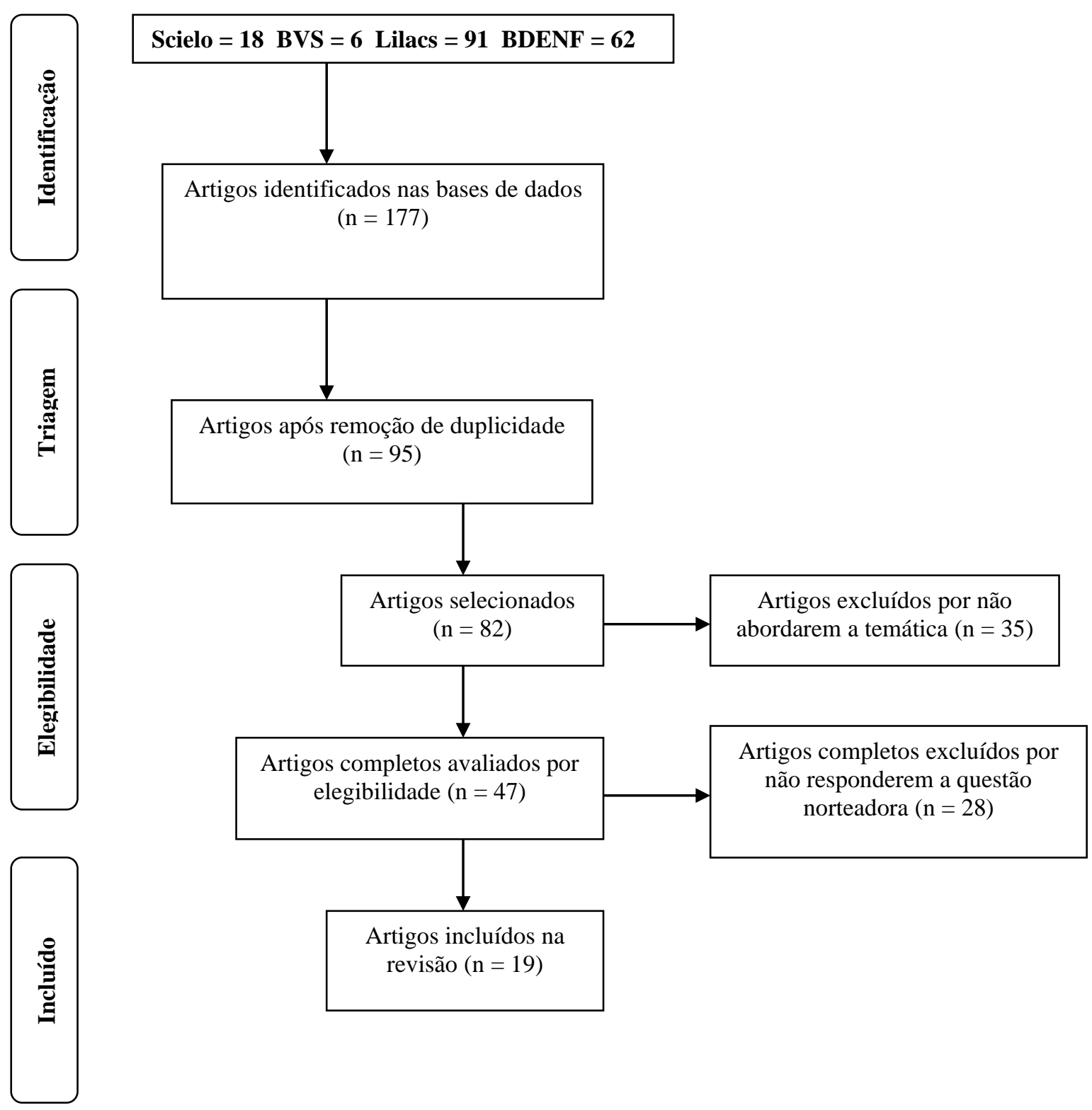

Fonte: Autores (2021).

\section{Resultados}

Com base na intercepção dos descritores e utilização dos critérios de inclusão foram selecionados a princípio 177 artigos científicos, depois de uma leitura minuciosa dos títulos e resumos, foram selecionados 19 artigos que atenderam exatamente a seleção da amostra pré estabelecida, provenientes das bases de dados. Com a finalidade de alcançar o objetivo do trabalho, após análise dos estudos foram definidas as seguintes categorias temáticas: capacitação dos profissionais de enfermagem da APS frente aos atendimentos de urgência e emergência e o uso do protocolo de acolhimento de classificação de risco na APS. O Quadro 1 abaixo expõe os artigos selecionados em ordem cronológica de publicação. 
Quadro 1- Síntese dos principais achados sobre a temática, Salvador - BA, 2021.

\begin{tabular}{|c|c|c|c|c|}
\hline $\begin{array}{c}\text { AUTOR } \\
\text { REVISTA }\end{array}$ & ANO & $\begin{array}{c}\text { METODOLOGI } \\
\text { A }\end{array}$ & OBJETIVO GERAL & CONCLUSÃO \\
\hline $\begin{array}{l}\text { Camerro, A, Alves, E. C., } \\
\text { Camerro, N. M. M. S., \& } \\
\text { Nogueira, L. D. P. } \\
\text { Revista Fafibe On-Line. }\end{array}$ & 2015 & $\begin{array}{l}\text { Revisão } \\
\text { bibliográfica. }\end{array}$ & $\begin{array}{l}\text { Levantar estudos sobre os } \\
\text { atendimentos realizados em } \\
\text { Serviços de Urgência e Emergência } \\
\text { do território Nacional. }\end{array}$ & $\begin{array}{l}\text { A falta de conhecimento dos usuários } \\
\text { ao utilizar os Serviços de Urgência e } \\
\text { Emergência, além da necessidade de } \\
\text { reforma nas Unidades de Saúde. }\end{array}$ \\
\hline $\begin{array}{l}\text { Marcondes, F. L., } \\
\text { Tavares, C. M. M., } \\
\text { Santos, G. S., Silva, T. } \\
\text { N., \& Silveira, P. G. } \\
\text { Revista Pró-UniverSUS. }\end{array}$ & 2015 & $\begin{array}{l}\text { Revisão } \\
\text { integrativa. }\end{array}$ & $\begin{array}{l}\text { Analisar a produção científica } \\
\text { nacional e internacional sobre a } \\
\text { capacitação de profissionais de } \\
\text { enfermagem na atenção primária a } \\
\text { saúde. }\end{array}$ & $\begin{array}{l}\text { Salienta-se que o processo de } \\
\text { capacitação é uma perspectiva de } \\
\text { aprendizagem no trabalho, devendo-se } \\
\text { investigar as reais demandas da unidade } \\
\text { e da equipe e a figura importante do } \\
\text { enfermeiro no processo de capacitação. }\end{array}$ \\
\hline $\begin{array}{l}\text { Nobrega, D. M., Bezerra, } \\
\text { A. L. D., Sousa, M. N. A. } \\
\text { Revista Eletrônica da } \\
\text { Fainor. }\end{array}$ & 2015 & $\begin{array}{l}\text { Estudo descritivo, } \\
\text { transversal. }\end{array}$ & $\begin{array}{l}\text { Analisar os conhecimentos, atitudes } \\
\text { e práticas de enfermeiros em } \\
\text { Urgência e Emergência na Atenção } \\
\text { Primária à Saúde. }\end{array}$ & $\begin{array}{l}\text { Faz-se necessário a promoção da } \\
\text { educação continuada e permanente na } \\
\text { área de Urgência e Emergência nos } \\
\text { serviços de saúde primários. }\end{array}$ \\
\hline $\begin{array}{l}\text { Smits, M., Peters, Y., } \\
\text { Broers, S., Keizer, E., } \\
\text { Wensing, M., \&Giesen, } \\
\text { P. BMC Family Practice. }\end{array}$ & 2015 & $\begin{array}{l}\text { Estudo } \\
\text { transversal. }\end{array}$ & $\begin{array}{l}\text { Examinar a associação entre as } \\
\text { características da prática clínica } \\
\text { diurna e o uso de cooperativas de } \\
\text { atenção à saúde fora do expediente. }\end{array}$ & $\begin{array}{l}\text { Além das características da população } \\
\text { de pacientes, as características } \\
\text { organizacionais das práticas clínicas } \\
\text { estãoassociados ao menor uso do } \\
\text { cuidados fora do expediente. }\end{array}$ \\
\hline $\begin{array}{l}\text { Traldi, M. C., Rabesco, } \\
\text { L., \& Fonseca, M. R. C. } \\
\text { C. REFACS. }\end{array}$ & 2016 & $\begin{array}{l}\text { Estudo } \\
\text { transversal. }\end{array}$ & $\begin{array}{l}\text { Avaliar o acolhimento dos serviços } \\
\text { de atenção básica a partir das fichas } \\
\text { classificadas como não urgência na } \\
\text { escala de Manchester. }\end{array}$ & $\begin{array}{l}\text { A capacidade de acolhimento de } \\
\text { crianças e adolescentes foi moderada, } \\
\text { com melhor desempenho das unidades } \\
\text { que atuam na Estratégia Saúde da } \\
\text { Família. }\end{array}$ \\
\hline $\begin{array}{l}\text { Baratieri, T., Paes de } \\
\text { Almeida, K., Lentsck, M. } \\
\text { H., \& Natal, S. Revista de } \\
\text { Saúde Pública do } \\
\text { Paraná. }\end{array}$ & 2017 & $\begin{array}{l}\text { Estudo descritivo, } \\
\text { qualitativo. }\end{array}$ & $\begin{array}{l}\text { Compreender os motivos que levam } \\
\text { os usuários a buscar as Unidades de } \\
\text { Pronto Atendimento (UPA) quando } \\
\text { o problema de saúde poderia ser } \\
\text { resolvido no âmbito da Atenção } \\
\text { Primária à Saúde (APS). }\end{array}$ & $\begin{array}{l}\text { As UPAs são vistas pela população } \\
\text { como mais resolutivas, havendo } \\
\text { necessidade de ordenar fluxos de } \\
\text { atendimento e educar/informar a } \\
\text { população sobre seu funcionamento. }\end{array}$ \\
\hline $\begin{array}{l}\text { Moreira, D. de A., } \\
\text { Tibães, H. B. B., Batista, } \\
\text { R. C. R., Cardoso, C. M. } \\
\text { L., \& Brito, M. J. M. } \\
\text { Texto \& contexto } \\
\text { enfermagem. }\end{array}$ & 2017 & $\begin{array}{l}\text { Estudo } \\
\text { qualitativo. }\end{array}$ & $\begin{array}{l}\text { Compreender ambiguidades e } \\
\text { desafios relacionados ao acesso, } \\
\text { após a implantação do Sistema de } \\
\text { Triagem de Manchester na atenção } \\
\text { primária à saúde. }\end{array}$ & $\begin{array}{l}\text { O estudo destaca a necessidade de } \\
\text { ampliar discussões sobre a legitimidade } \\
\text { do Sistema de Triagem de Manchester } \\
\text { na atenção primária à saúde, tendo em } \\
\text { vista ambiguidades e desafios referentes } \\
\text { à garantia de acesso neste espaço de } \\
\text { produção do cuidado. }\end{array}$ \\
\hline $\begin{array}{l}\text { Rossato, K., Real, V. R., } \\
\text { Oliveira, G. B. de, } \\
\text { \&Araujo, C. D. C. de. } \\
\text { Revista de Enfermagem } \\
\text { da UFSM. }\end{array}$ & 2018 & $\begin{array}{l}\text { Estudo de campo, } \\
\text { descritivo. }\end{array}$ & $\begin{array}{l}\text { Conhecer a opinião da equipe de } \\
\text { enfermagem de três Estratégias de } \\
\text { saúde da Família de um município } \\
\text { do interior do Rio Grande do Sul } \\
\text { acerca do Acolhimento com } \\
\text { Classificação de Risco na Estratégia } \\
\text { de Saúde da Família. }\end{array}$ & $\begin{array}{l}\text { Constatou-se que muitos dos } \\
\text { profissionais encontraram dificuldades } \\
\text { em realizar o acolhimento com } \\
\text { classificação de risco, pois, além do } \\
\text { processo de trabalho estar centrado no } \\
\text { saber médico, os profissionais não } \\
\text { possuem capacitação e um protocolo } \\
\text { direcionador para realização da } \\
\text { classificação do risco. }\end{array}$ \\
\hline $\begin{array}{l}\text { Silveira, M. A., Lima, P. } \\
\text { V. S., Cavalcante, A. B., } \\
\text { Trindade, L. S. da, } \\
\text { Catapreta, A. A., \& } \\
\text { Barros, A. M. M. S. } \\
\text { Ciências Biológicas E Da } \\
\text { Saúde-UNIT. }\end{array}$ & 2018 & $\begin{array}{l}\text { Revisão } \\
\text { bibliográfica }\end{array}$ & $\begin{array}{l}\text { Compreender o acolhimento e a } \\
\text { classificação de risco no Sistema } \\
\text { Único de Saúde. }\end{array}$ & $\begin{array}{l}\text { A elevada procura por atendimento } \\
\text { nesses serviços } \\
\text { desorganização na prestação da } \\
\text { assistência, o que resulta na } \\
\text { imprescindível instalação de Protocolos } \\
\text { de Acolhimento com Avaliação e } \\
\text { Classificação de Risco. }\end{array}$ \\
\hline $\begin{array}{l}\text { Coloni, C. S. M. Agencia } \\
\text { USP de Gestão da } \\
\text { Informação Acadêmica. }\end{array}$ & 2019 & Estudo descritivo. & $\begin{array}{l}\text { Identificar as necessidades de } \\
\text { aprendizagem sobre o acolhimento } \\
\text { com classificação de risco da } \\
\text { demanda espontânea na Atenção } \\
\text { primária à Saúde. }\end{array}$ & $\begin{array}{l}\text { O reconhecimento das limitações para } \\
\text { implementação do protocolo, requer a } \\
\text { sistematização de processo de educação } \\
\text { permanente em saúde para } \\
\text { problematizar a realidade da unidade de } \\
\text { saúde e proporcionar a capacitação aos }\end{array}$ \\
\hline
\end{tabular}




\begin{tabular}{|c|c|c|c|c|}
\hline & & & & $\begin{array}{l}\text { enfermeiros que são os responsáveis } \\
\text { legais pelo protocolo de Manchester. }\end{array}$ \\
\hline $\begin{array}{l}\text { Garcia, J. } \quad \text { A.C. } \quad \text { L. } \\
\text { Universidade } \\
\text { Federal de } \\
\text { Alagoas. }\end{array}$ & 2019 & $\begin{array}{l}\text { Estudo } \\
\text { exploratório, } \\
\text { descritivo }\end{array}$ & $\begin{array}{l}\text { Analisar a percepção dos } \\
\text { enfermeiros sobre sua atuação em } \\
\text { casos de urgência e emergência na } \\
\text { Atenção Básica. }\end{array}$ & $\begin{array}{l}\text { A capacitação profissional deverá vir } \\
\text { acompanhada de melhorias na } \\
\text { infraestrutura material e física para } \\
\text { atender os casos de urgência e } \\
\text { emergência nessas unidades }\end{array}$ \\
\hline $\begin{array}{l}\text { Hermida, P. M. V., } \\
\text { Nascimento, E. R. P. do, } \\
\text { Echevarría-Guanilo, M. } \\
\text { E., Andrade, S. R. de, \& } \\
\text { Ortiga, Â. M. B. Revista } \\
\text { Brasileira } \\
\text { Enfermagem. }\end{array}$ & 2019 & $\begin{array}{l}\text { Estudo descritivo, } \\
\text { qualitativo }\end{array}$ & $\begin{array}{l}\text { Descrever as facilidades e } \\
\text { dificuldades da contra-referência de } \\
\text { uma Unidade de Pronto } \\
\text { Atendimento do estado de Santa } \\
\text { Catarina. }\end{array}$ & $\begin{array}{l}\text { As dificuldades evidenciadas indicam } \\
\text { expressivos desafios do sistema de } \\
\text { saúde local na busca da integração entre } \\
\text { os pontos de atenção às urgências. }\end{array}$ \\
\hline 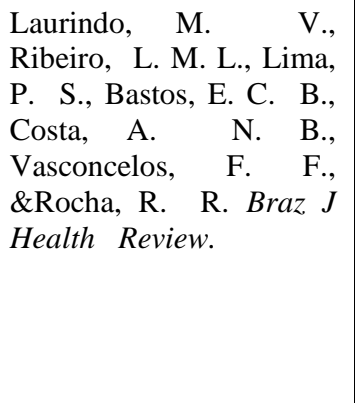 & 2019 & $\begin{array}{l}\text { Revisão } \\
\text { integrativa. }\end{array}$ & $\begin{array}{l}\text { Delinear os estudos que abordem o } \\
\text { atendimento de urgência e } \\
\text { emergência na unidade básica de } \\
\text { saúde e avaliar a importância de } \\
\text { adaptar as mesmas para o } \\
\text { atendimento de urgências e } \\
\text { emergências de menor } \\
\text { complexidade. }\end{array}$ & $\begin{array}{l}\text { O atendimento na atenção primária á } \\
\text { saúde em relação às urgências e } \\
\text { emergências dependem de vários } \\
\text { fatores, que englobam a rede de atenção } \\
\text { às urgências devendo fluir em todos os } \\
\text { níveis do SUS. Junto a isso, a } \\
\text { capacitação dos profissionais e a } \\
\text { adequação do espaço físico nas } \\
\text { unidades básica de saúde são } \\
\text { fundamentais para atenção e agilidade } \\
\text { nos atendimentos aos casos graves. }\end{array}$ \\
\hline $\begin{array}{l}\text { Peiter, C. C., Santos, J. L. } \\
\text { G. dos, Lanzoni, G. M. } \\
\text { de M., Mello, A. L. S. F. } \\
\text { de, Costa, M. F. B. N. A. } \\
\text { da, \& Andrade, S. R. de. } \\
\text { Escola Anna Nery. }\end{array}$ & 2019 & $\begin{array}{l}\text { Estudo } \\
\text { documental. }\end{array}$ & $\begin{array}{l}\text { Analisar a tendência de } \\
\text { investigação de teses e dissertações } \\
\text { brasileiras da área da saúde sobre o } \\
\text { tema Redes de } \\
\text { Atenção à Saúde }\end{array}$ & $\begin{array}{l}\text { São tendências da produção do } \\
\text { conhecimento o estudo da atenção } \\
\text { primária como ordenadora da rede e sua } \\
\text { relação com os demais níveis, a } \\
\text { formação dos profissionais para atuação } \\
\text { articulada e integrada, e a educação } \\
\text { permanente em saúde, com foco na } \\
\text { gestão das redes de atenção à saúde. }\end{array}$ \\
\hline $\begin{array}{l}\text { Pereira, S. } \quad \text { B., \& } \\
\text { Brasileiro, M. E. Gestão } \\
\text { \& Tecnologia } \\
\text { Daculdade } \\
\text { Delta. }\end{array}$ & 2019 & $\begin{array}{l}\text { Revisão } \\
\text { integrativa. }\end{array}$ & $\begin{array}{l}\text { Descrever sobre acolhimento e } \\
\text { vinculo relatar sobre percepção dos } \\
\text { profissionais sobre o acolhimento e } \\
\text { demonstrar os fatores indicados } \\
\text { como facilitadores e dificultadores } \\
\text { para a prática do acolhimento. }\end{array}$ & $\begin{array}{l}\text { A classificação de risco é apontada } \\
\text { como um instrumento de organização, } \\
\text { principalmente para aquelas unidades } \\
\text { de Saúde da Família em que a } \\
\text { quantidade de consultas ofertadas é } \\
\text { inferior à demanda do dia. Com isto, o } \\
\text { enfermeiro considera que o atendimento } \\
\text { realizado pelo critério de prioridade e } \\
\text { não pela ordem de chegada torna a } \\
\text { assistência mais humanizada e } \\
\text { acolhedora. }\end{array}$ \\
\hline $\begin{array}{l}\text { Sade, P. M. C., Peres, A. } \\
\text { M., Brusamarello, T., } \\
\text { Das Mercês, N. N. A., } \\
\text { Wolff, L. D. G., } \\
\text { \&Lowen, I. M. } \quad \text { V. } \\
\text { Cogitare Enfermagem. }\end{array}$ & 2019 & $\begin{array}{l}\text { Pesquisa } \\
\text { documental. }\end{array}$ & $\begin{array}{l}\text { Analisar demandas de educação } \\
\text { permanente da equipe de } \\
\text { enfermagem de um hospital público } \\
\text { de ensino do sul do Brasil. }\end{array}$ & $\begin{array}{l}\text { A análise documental de tais demandas } \\
\text { subsidia o planejamento e e } \\
\text { aperfeiçoamento de propostas de } \\
\text { educação permanente em enfermagem. }\end{array}$ \\
\hline $\begin{array}{l}\text { Damaceno, A. N., Lima, } \\
\text { M. A. D. da S., Pucci, V. } \\
\text { R., \&Weiller, T. H. } \\
\text { Revista de Enfermagem } \\
\text { da UFSM. }\end{array}$ & 2020 & Estudo reflexivo. & $\begin{array}{l}\text { Discutir e refletir sobre as Redes de } \\
\text { Atenção à Saúde, seus conceitos } \\
\text { teóricos e marcos legais nas } \\
\text { políticas de saúde. }\end{array}$ & $\begin{array}{l}\text { A conformação de Redes de Atenção à } \\
\text { Saúde colabora para superação do } \\
\text { modelo verticalizado. A estruturação é } \\
\text { pautada com base no perfil } \\
\text { epidemiológico e colabora para } \\
\text { melhoria da atenção prestada, com } \\
\text { superação de lacunas assistenciais e } \\
\text { reduçâo de custos. }\end{array}$ \\
\hline $\begin{array}{l}\text { Freitas, T. C. C., Ferreira } \\
\text { Moreira, G. G., Aquino, } \\
\text { J. M., Lacerda, K. P. C., } \\
\text { da Silva, R., Araújo de } \\
\text { Jesus, A. P. G., Fonseca, } \\
\text { A. C. S., Gonçalves, K. }\end{array}$ & 2020 & $\begin{array}{l}\text { Revisão } \\
\text { integrativa }\end{array}$ & $\begin{array}{l}\text { Identificar a atuação dos } \\
\text { profissionais da atenção primária a } \\
\text { saúde frente às urgências e } \\
\text { emergências à luz da revisão } \\
\text { integrativa da literatura. }\end{array}$ & $\begin{array}{l}\text { Para que a atenção básica seja } \\
\text { participante e atuante efetiva na rede de } \\
\text { urgências, cabe aos gestores a inclusão } \\
\text { deste tema em conferências municipais } \\
\text { de saúde e treinamento para que toda a } \\
\text { equipe possa prestar um atendimento }\end{array}$ \\
\hline
\end{tabular}




\begin{tabular}{|l|l|l|l|l|}
\hline $\begin{array}{l}\text { B., Botelho, A. S., \& } \\
\text { Gonçalves, M. D. Revista } \\
\text { Eletrônica Acervo Saúde. }\end{array}$ & & & $\begin{array}{l}\text { efetivo ao usuário em situação de } \\
\text { urgência e emergência. }\end{array}$ \\
\hline $\begin{array}{l}\text { Silveira, A. Universidade } \\
\text { Vale do Rio dos Sinos. }\end{array}$ & 2020 & $\begin{array}{l}\text { Pesquisa } \\
\text { documental }\end{array}$ & $\begin{array}{l}\text { Construir um fluxograma para o } \\
\text { acolhimento de situações de } \\
\text { Urgência e Emergência na Atenção } \\
\text { Primária à Saúde do Município de } \\
\text { Gramado/RS. }\end{array}$ & $\begin{array}{l}\text { Cosntatou-se que não há padronização } \\
\text { para os atendimentos por demanda } \\
\text { como nem tom do município, assim } \\
\text { realizam o ACCR. }\end{array}$ \\
\hline
\end{tabular}

Fonte: Autores (2021).

\section{Capacitação dos profissionais de enfermagem da atenção primária frente aos atendimentos de urgência e emergência}

A Atenção Primária à Saúde (APS) tem como principal atribuição aplicar a promoção e a prevenção à saúde, a equipe de enfermagem deve está capacitada para possíveis situações de emergência, já que os usuários da área de abrangência apresentam morbidade que podem levar a casos de emergência.

O conceito de Urgência e Emergência (UE) segundo Laurindo et al. (2019), precisam está bem definidos, tanto pela comunidade quanto pelos profissionais de saúde, pois o conceito pode divergir podendo interferir na procura e na assistência. Para os profissionais de saúde a urgência está associada ao tempo, proporcional ao prognóstico, porém, para o usuário está relacionada à interrupção da vida.

Segundo Peiter et al. (2019) no Brasil, o Sistema Único de Saúde (SUS), organizado por meio do estabelecimento das Redes de Atenção à Saúde (RAS), busca qualificar a atenção por meio da ampliação do acesso e longitudinalidade do cuidado, tendo como objetivo o alcance da integralidade. Nas RAS, a população, a estrutura operacional e os modelos de atenção devem ser definidos de modo a responder prontamente aos eventos agudos, como também manejar as condições crônicas de saúde.

A responsabilidade da APS nas ações de coordenação de cuidados e garantia da continuidade da atenção, tem sido objeto de discussão e reflexão no campo da saúde. Para o momento sanitário atual, com o crescimento da incidência de doenças crônicas e alteração na estrutura etária da população, revela-se necessária uma resposta dos serviços de saúde para a organização de um sistema de referência responsável, eficiente e integrado (Damaceno, Lima, Pucci, \&Weiller, 2020).

Para se exercer um atendimento de excelência, ainda existem alguns obstáculos que precisam ser sanados na esfera de gestão da APS como referência universal ao usuário. No que se refere às demandas programadas, agendamentos, demanda espontânea e, especialmente, nas UE que representam as situações nas quais os usuários chegam ao serviço apresentando desestabilização de quadros mórbidos pré-existentes ou situações de novos agravos à saúde, ainda existe uma série de dificuldades na prestação de uma assistência eficaz ao paciente (Freitas et al., 2020).

Os profissionais de enfermagem da APS devem ter qualificação e competência para atender as demandas de UE, visto que a unidade de saúde é a primeira porta de entrada na RAS. A negação de atendimento e a referência inadequada para outro serviço de saúde são obstáculos enfrentados em todos os níveis de atenção. Os usuários do serviço procuram a unidade por já ser acompanhados pelos profissionais, e por oferecer uma assistência de saúde rápida, efetiva, e próximo da sua residência.

Conforme (2019) a escassez de recursos humanos e a falta de capacitação são algumas das barreiras gerências enfrentadas. $\mathrm{O}$ desconhecimento sobre os protocolos ainda suscita dúvidas, fazendo com que os profissionais percebam a necessidade de aprofundar os aspectos teórico-práticos da clínica, para realizar a classificação com segurança e mitigar os riscos de uma tomada de decisão equivocada.

Somente o Caderno de Acolhimento à Demanda Espontânea n ${ }^{\circ}$ 28, composta por dois volumes apresenta algumas instruções que podem ajudar o profissional da APS em situações de UE, apresentando orientações básicas sobre acolhimento da demanda espontânea, abrangendo os principais aspectos que envolvem a rotina (Garcia, 2019).

Para manter o controle dos atendimentos disponibilizados, é muito importante que se tenha uma gestão eficaz e um conhecimento referente às condutas nos atendimentos de UE. Conduzir a demanda de acordo com as competências clínicas 
contribui na redução dos problemas emergenciais através do direcionamento dos pacientes, seja pela estruturação da rede de encaminhamentos ou pelo planejamento assistencial (Camerro, Alves, Camerro, \& Nogueira, 2015).

De acordo com Smits et al. (2015), os profissionais de enfermagem devem estar preparados para identificar, através da avaliação dos sinais e sintomas de cada faixa etária, os sinais de gravidade, a impressão inicial do paciente em UE possibilitando assim o reconhecimento rápido da instabilidade fisiológica, prestando um atendimento eficaz e seguro.

A capacitação dos profissionais torna-se pertinente, frente à importância da afinidade com as práticas educativas que caracterizam sua formação, sendo assim uma ferramenta para aperfeiçoamento da assistência e na contribuição da sua valorização profissional.

Segundo Marcondes, Tavares, Santos, Silva e Silveira (2015) é fundamental que os profissionais de enfermagem se mantenham capacitados e atualizados para que além de fornecer uma assistência de qualidade, eles preencham lacunas de sua formação levando-se em conta o mundo globalizado e suas constantes transformações.

Para Garcia (2019) a educação permanente em saúde (EPS) surge como uma estratégia fundamental para enriquecer e ampliar a prática profissional, sobretudo, com o avanço de tecnologias e novas práticas de cuidados. Sua aprendizagem se dá pela problematização da realidade, desenvolvendo propostas visando modificar as práticas de trabalho, de acordo com a atividade profissional. Portanto a EPS visam mudanças nas estratégias organizacionais para qualificar as novas demandas no processo de trabalho da enfermagem (Sade, 2019).

Dessa forma, o atendimento adequado às UE depende de vários fatores, como: infraestrutura local, sistema de central de regulação dos casos e da capacitação de recursos humanos. Portanto, a capacitação dos profissionais e a adequação do espaço físico nas APS são fundamentais para a atenção aos casos urgentes e emergentes (Laurindo et al., 2019).

Diante do exposto é imprescindível a capacitação profissional e o cumprimento dos protocolos clínicos, resolutividade dos encaminhamentos de referência e contra-referência, e adaptação dos instrumentos materiais e ambientais para eficácia da assistência na abordagem primária.

\section{Uso do protocolo de acolhimento de classificação de risco na atenção primária à saúde}

As sobrecargas dos serviços hospitalares e das unidades de pronto atendimento por pacientes com queixas próprias da APS, justificam o congestionamento desses setores. Sendo assim, a maior parte dos atendimentos de UE caracteriza agravos que podem ser sanados ambulatorialmente, reduzindo o quantitativo de atendimentos hospitalares.

Conforme propõe a Rede de Atenção às Urgências e Emergências (RUE), é nesse contexto que se discute acolhimento e classificação de risco dos usuários, a diferenciação da demanda, o processo de trabalho das equipes de saúde, o cuidado e as condutas frente às necessidades e queixas, cabendo à APS ser o ponto de atenção à saúde norteador do fluxo do usuário nessa rede (Baratiere, Almeida, Lentsck, \& Natal, 2017).

As políticas públicas de saúde e de humanização referem que acolher na APS é uma maneira objetiva de identificar, reconhecer e intervir sobre as demandas que chegam do usuário, aceitando o fato de que este também define necessidades de saúde em formas e graus variados. Com o objetivo de reduzir o tempo de espera e equacionar o atendimento, os serviços públicos passaram a adotar medidas de acolhimento normatizadas em um protocolo para classificação de risco nos serviços de pronto atendimento (Traldi, Rabesco, \& Fonseca, 2016).

O Acolhimento com Classificação de Risco (ACCR) surgiu com o intuito de mudar a lógica do atendimento, priorizando o agravo à saúde e o sofrimento do usuário, e não a ordem de chegada ao serviço de saúde, com o objetivo de organizar as filas de espera para os atendimentos, além de informar o tempo de espera e retornar as informações aos familiares, além de promover a ampla informação sobre o serviço aos usuários (Pereira \& Brasileiro, 2019). 
Todos os profissionais da unidade atuam no ACCR, começando pela recepção, sendo o contato inicial do paciente, continuando a triagem com os profissionais de enfermagem e por último com o médico.

Segundo Silveira et al. (2018) para que o enfermeiro atue na Classificação de Risco é necessário o desenvolvimento de habilidades e competências que estimulem o raciocínio crítico e o atendimento específico, como observação do paciente, escuta qualificada, tomada de decisão, priorização do atendimento de acordo com as necessidades dos usuários, bem como delegar funções, a fim de proporcionar uma assistência eficiente, eficaz e efetiva.

Nesta circunstância, a classificação de risco caracteriza-se como parte crucial do acolhimento. Porém, faz-se necessário legitimar e qualificar os profissionais de enfermagem, com o intuito de fortalecer essa estratégia que auxilia na reestruturação das redes de cuidado em saúde e possibilitando que os pacientes sejam atendidos com resolutividade e humanização.

Quando o usuário com queixas agudas procura pelo atendimento e sua demanda ultrapassa a capacidade resolutiva da APS, a referência do usuário para outros serviços de saúde deve ser assegurada conforme a proposta da RAS para atender à demanda com responsabilidade (Moreira, Tibães, Batista, Cardoso, \& Brito, 2017).

O acolhimento realizado ao usuário na Atenção Primária pode ser entendido como uma estratégia de comunicação que facilita a contra-referência entre a Unidade de Pronto Atendimento (UPA) e a Unidade de Saúde da Família (USF). Nesse sentido, o acolhimento se torna um espaço potente para humanização dos serviços de saúde (Hermida, Nascimento, Guanilo, Andrade, \& Ortiga, 2019).

A implantação do ACCR através do Sistema de Triagem de Manchester (STM) nas unidades da APS auxilia na padronização dos critérios de avaliação, assegurando um atendimento de demanda espontânea em tempo reduzido, além de integralizar os serviços de saúde e diminuindo a desintegração da rede assistencial.

O STM classifica o paciente em cinco níveis, utilizando um sistema de cores para organizar o tempo máximo de espera para o atendimento adequado. A partir dos sinais e sintomas apresentados pelo paciente, o profissional responsável direciona as prioridades organizando os pacientes para o atendimento de acordo com os níveis de urgência (Coloni, 2019).

Tabela 1 - Classificação de Risco estabelecido pelo Sistema de Triagem de Manchester.

\begin{tabular}{llll}
\hline Número & Nome & Cor & Tempo-resposta máximo (min) \\
\hline 1 & Emergente & Vermelho & 0 \\
2 & Muito urgente & Laranja & 10 \\
3 & Urgente & Amarelo & 60 \\
4 & Pouco urgente & Verde & 120 \\
5 & Não urgente & Azul & 240 \\
\hline
\end{tabular}

Fonte: Grupo Brasileiro de Classificação de Risco (2015).

Cada fluxograma contém os discriminadores, definidos previamente para garantir a uniformidade, a compreensão e a aplicabilidade dos conceitos que orientarão a coleta e análise de informações para a definição da prioridade clínica do paciente (Grupo Brasileiro de Classificação de Risco [GBCR], 2015).

Para que o atendimento ocorra de forma correta, é preciso que os profissionais de enfermagem tenham o conhecimento sobre o acolhimento, e que este resulta em escutar, valorizar e identificar os riscos e necessidades dos usuários, de modo a ofertar um encaminhamento adequado. A avaliação é realizada através da consulta de enfermagem durante o acolhimento, após a escuta qualificada, anamnese e exame físico, quando é decidida a conduta adequada para o usuário (Rossato, 2018). 


\section{Discussão}

A população do estudo foi composta por 05 técnicos de enfermagem e 03 enfermeiras atuantes na Unidade de Saúde da Família situada no Subúrbio Ferroviário de Salvador-Bahia. A pesquisa foi realizada por $100 \%$ dos profissionais de enfermagem sendo a maioria composta pelo sexo feminino (87,5\%; $n=7)$, com idade superior aos 30 anos (100\%; $n=8)$, sendo $(62,5 \% ; n=5)$ técnicos de enfermagem e $(37,5 \% ; n=3)$ enfermeiras e $(62,5 \% ; n=5)$ com mais de 5 anos de atuação na APS, todos informaram que já trabalharam em emergência.

Questionou-se aos participantes do estudo se eles realizaram curso de capacitação em urgência e emergência, somente $(75 \% ; n=6)$ dos profissionais participaram de cursos e $(25 \% ; n=2)$ não, dos capacitados $(62,5 \% ; n=5)$ fizeram curso por conta própria e somente $(37,5 \% ; n=3)$ fizeram cursos oferecido pela Secretaria Municipal de Saúde.

Dessa forma, o estudo demonstra a necessidade da realização de cursos de capacitação para todos os profissionais de enfermagem da APS, visto que a falta de informação sobre a temática pode afetar a assistência recomendada pela atenção primária. O profissional deve está apto para prestar uma assistência adequada, para que consiga reconhecer as situações de urgência e dá resolução aos casos.

Observou-se que apenas $(50 \% ; n=4)$ dos profissionais se sentem aptos para atender casos de urgência e emergência, isso tem relação com o fato desses profissionais terem vínculo empregatício na área hospitalar. É imprescindível que o profissional tenha conhecimento em várias áreas da saúde, já que todo usuário do ACCR pode exigir um atendimento momentâneo.

Com essa análise percebemos que uma parte dos profissionais se aflige com a falta de segurança e a carência da prática e experiência envolvendo situações de urgência e emergência.

Outro questionamento foi se eles tinham informação sobre a Escala de Manchester, (87,5\%; n=7) já ouviram falar, porém, nenhum profissional utiliza, $(100 \% ; n=8)$ atende os pacientes por ordem de chegada. A não utilização pode está ligada a falta de treinamento dos profissionais, escassez de recursos materiais e de infraestrutura adequada para o cuidado.

Este dado nos faz considerar uma limitação dos profissionais com relação à percepção e aplicabilidade do protocolo, demonstrando a necessidade de respaldo advindo das autoridades, criando protocolos de orientação em cooperação com a educação permanente.

\section{Considerações Finais}

Diante do número crescente da demanda de atendimentos emergenciais na atenção primária á saúde, e da necessidade de um nível de instrução e de conhecimento dos profissionais em relação aos protocolos de acolhimento e excelência no desfecho clínico, ative-me a desenvolver um estudo abordando a temática.

No decorrer do desenvolvimento do estudo, buscou-se responder as questões voltadas ao nível de conhecimento dos profissionais da atenção primária, em relação aos cuidados voltados aos pacientes no aspecto emergencial, o acolhimento de forma humanizada e a criticidade conforme o protocolo de Manchester.

Entretanto ao iniciar a pesquisa a demanda pela temática mostrou-se com pouca abordagem de estudos, mesmo variando os descritores. Ao realizar uma seleção, resultou de poucos artigos científicos disponíveis que descrevesse o tema.

Por fim considerando como um estudo relevante de dimensão direcionada, o método de reestruturação da Atenção Primária à Saúde, qualificação da assistência e capacitação dos profissionais de enfermagem, resulta-se em pesquisa de grande impacto no novo modelo de educação e saúde para os profissionais e um dimensionamento no atendimento com acolhimento de risco ao usuário da unidade de saúde primária. 


\section{Referências}

Baratieri, T., Paes de Almeida, K., Lentsck, M. H., \& Natal, S. (2017). Percepções de usuários atendidos em um pronto atendimento: olhar sobre a atenção primária à saúde. Espaço para a Saúde - Revista de Saúde Pública do Paraná, 18(1), 54.

Camerro, A, Alves, E. C., Camerro, N. M. M. S., \& Nogueira, L. D. P. (2015). Perfil do atendimento de serviços de urgência e emergência. Revista Fafibe OnLine, 8(1), 515-524.

Coloni, C. S. M. (2019). Acolhimento com classificação de risco da demanda espontânea: as necessidades de aprendizagem de enfermeiros da atenção primária à saúde. Universidade de Sao Paulo, Agencia USP de Gestao da Informação Acadêmica (AGUIA).

Damaceno, A. N., Lima, M. A. D. da S., Pucci, V. R., \&Weiller, T. H. (2020). Redes de atenção à saúde: uma estratégia para integração dos sistemas de saúde. Revista de Enfermagem da UFSM, 10, e14.

Freitas, T. C. C., Ferreira Moreira, G. G., Aquino, J. M., Lacerda, K. P. C., da Silva, R., Araújo de Jesus, A. P. G., Fonseca, A. C. S., Gonçalves, K. B., Botelho, A. S., \& Gonçalves, M. D. (2020). A Atenção Primária como parte integrante da rede de atendimento as Urgências e Emergências: à luz da literatura. Revista Eletrônica Acervo Saúde, (38), e2881. https://doi.org/10.25248/reas.e2881.2020

Garcia, J. A.C. L. (2019). Atuação em urgência e emergência na atenção básica: percepção dos enfermeiros (Dissertação de Mestrado). Universidade Federal de Alagoas, Maceió, AL, Brasil.

Grupo Brasileiro de Classificação de Risco. (2015) Diretrizes para implementação do Sistema de Manchester de Classificação de Risco nos pontos de atenção às urgências e emergências. Belo Horizonte.

Hermida, P. M. V., Nascimento, E. R. P. do., Echevarría-Guanilo, M. E., Andrade, S. R. de., \& Ortiga, Â. M. B. (2019). Counter-referral in Emergency Care Units: discourse of the collective speech. Revista Brasileira de Enfermagem, 72(suppl 1), 143-150.

Laurindo, M. V., Ribeiro, L. M. L., Lima, P. S., Bastos, E. C. B., Costa, A. N. B., Vasconcelos, F. F., \& Rocha R. R. (2019). A importância de adaptar as unidades básicas de saúde para o atendimento de urgências e emergências de menor complexidade. Braz J Health Review, 2(3), 1688-1709. http://www.brazilianjournals.com/index.php/BJHR/article/view/1434/1551

Marcondes, F. L., Tavares, C. M. M., Santos, G. S., Silva, T. N., \& Silveira, P. G. (2015). Capacitação profissional de enfermagem na atenção primária à saúde: Revisão integrativa. Revista Pró-UniverSUS, 06(3), 09-15.

Ministério da Saúde. (2014). Humaniza SUS:acolhimento com avaliação e classificação de risco: um paradigma ético- estético no fazer em saúde. Brasília.

Moreira, D. de A., Tibães, H. B. B., Batista, R. C. R., Cardoso, C. M. L., \& Brito, M. J. M. (2017). Manchester Triage System in primary health care: Ambiguities and challenges related to access. Texto \& contexto enfermagem, 26(2). https://doi.org/10.1590/0104-07072017005970015

Nobrega, D. M., Bezerra, A. L. D., \& Sousa, M. N. A. (2015). Conhecimentos, Atitudes e Práticas em Urgência e Emergência na Atenção Primária à Saúde. Revista Eletrônica da Fainor, 8(2), 141-157.

Peiter, C. C., Santos, J. L. G. dos., Lanzoni, G. M. de M., Mello, A. L. S. F. de., Costa, M. F. B. N. A. da., \& Andrade, S. R. de. (2019). Healthcare networks: trends of knowledge development in Brazil. Escola Anna Nery, 23(1). https://doi.org/10.1590/2177-9465-ean-2018-0214

Pereira, S. B., \& Brasileiro, M. E. (2019). Acolhimento com classificação de risco na atenção primária à saúde: revisão integrativa. Gestão \& Tecnologia Faculdade Delta, 2(29).

Rossato, K., Real, V. R., Oliveira, G. B. de, \&Araujo, C. D. C. de. (2018). Acolhimento com classificação de risco na Estratégia de Saúde da Família: percepção da equipe de enfermagem. Revista de Enfermagem da UFSM, 8(1), 144.

Sade, P. M. C., Peres, A. M., Brusamarello, T., Das Mercês, N. N. A., Wolff, L. D. G., \&Lowen, I. M. V. (2019). Demandasde Educação Permanentede Enfermagemem Hospitalde Ensino. Cogitare Enfermagem, 24. https://doi.org/10.5380/ce.v24i0.57130

Silveira, A. (2020). Fluxograma de acolhimento das situações de urgência e emergência na atenção primária em saúde (Dissertação de Mestrado). Universidade Vale do Rio dos Sinos.

Silveira, M. A., Lima, P. V. S., Cavalcante, A. B., Trindade, L. S. da., Catapreta, A. A., \& Barros, A. M. M. S. (2018). A Aplicabilidadeda Classificaçãode Risco no Sistema Único de Saúde: Revisão Bibliográfica. Caderno De Graduação - Ciências Biológicas E Da Saúde - UNIT - SERGIPE, 4(3), 11.

Smits, M., Peters, Y., Broers, S., Keizer, E., Wensing, M., \& Giesen, P. (2015). Association between general practice characteristics and use of out-of-hours GP cooperatives. BMC Family Practice, 16(1), 52.

Traldi, M. C., Rabesco, L., \& Fonseca, M. R. C. C. (2016). Avaliação da capacidade de acolhimento da demanda espontânea nos serviços de atenção básica. REFACS, 4(2), 107-118. 